\title{
INDEPENDENCE AND CONSISTENCY PROOFS IN QUADRATIC FORM THEORY
}

\author{
JAMES E. BAUMGARTNER AND OTMAR SPINAS
}

Introduction. We consider the following properties of uncountable-dimensional quadratic spaces $(E, \Phi)$ :

(*) For all subspaces $U \subseteq E$ of infinite dimension: $\operatorname{dim} U^{\perp}<\operatorname{dim} E$.

(**) For all subspaces $U \subseteq E$ of infinite dimension: $\operatorname{dim} U^{\perp} \leq \aleph_{0}$.

Spaces of countable dimension are the orthogonal sum of straight lines and planes, so they cannot have $(*)$, but $(* *)$ is trivially satisfied.

These properties have been considered first in $[\mathrm{G} / \mathrm{O}]$ in the process of investigating the orthogonal group of quadratic spaces. It has been shown there (in ZFC) that over arbitrary uncountable fields $(* *)$-spaces of uncountable dimension exist.

In $[\mathrm{B} / \mathrm{G}],(* *)$-spaces of dimension $\aleph_{1}($ so $(*)=(* *))$ have been constructed over arbitrary finite or countable fields. But this could be done only under the assumption that the continuum hypothesis $(\mathrm{CH})$ holds in the underlying set theory.

In Chapter 3 of [B/G], Jensen's principle $\diamond$ has been used to construct an $\aleph_{1}$ dimensional space $(E, \Phi)$ without any $\perp$-dense orthogonal system, i.e. for any subspace $U$ spanned by an orthogonal basis ( $U$ "diagonal") we have $U^{\perp \perp} \neq E$. Some time later $\mathrm{H}$. Gross realized that in their construction only $\mathrm{CH}$ is needed instead of $\diamond$.

Following their method of proof, in $\S 1$ we generalize all the results of $[\mathrm{B} / \mathrm{G}]$ by assuming Martin's axiom (MA) instead of $\mathrm{CH}$. We obtain (*)-spaces and spaces without any dense orthogonal system of dimension $c$ (continuum).

In $\$ 2$ we show that the existence of uncountable-dimensional (**)-spaces over finite or countable fields is independent of ZFC. Namely, in models for ZFC + $\mathfrak{p}>\aleph_{1}$ such spaces cannot exist. Here $\mathfrak{p}$ is one of the so-called cardinal invariants of the continuum; these are uncountable cardinals $\leq \mathrm{c}$. In ZFC it cannot be decided where they lie exactly. As a consequence we get that the (*)-spaces in models for ZFC $+\neg \mathrm{CH}+\mathrm{MA}$ in $\$ 1$ are not $(* *)$, for MA implies $\mathfrak{p}=\mathfrak{c}$. By using Sacks forcing, we show that the existence of $(*)$-spaces over finite or countable fields, at least if of dimension $\mathfrak{c}$, is independent, too. There exists a model for $\mathfrak{b}=\mathfrak{p}=\mathfrak{\aleph}_{1}+\neg \mathrm{CH}$ where such spaces do not exist.

In $\$ 3$ we look at consistency in the other direction. We show that $\mathrm{CH}$ is not necessary for the existence of $(* *)$-spaces. By Cohen forcing, we prove that there exist

Received July 23, 1990; revised November 14, 1990.

(C) 1991, Association for Symbolic Logic $0022-4812 / 91 / 5604-0003 / \$ 02.70$ 
(**)-spaces of dimension $c$ over arbitrary finite or countable fields in models for $\mathrm{ZFC}+\neg \mathrm{CH} . \mathrm{c}$ is the maximal possible dimension of such spaces, for it is not difficult to see that a (**)-space $E$ over $k$ satisfies $\operatorname{dim} E \leq|k|^{N_{0}}$. So this result cannot be improved.

The spaces $(E, \Phi)$ which we construct have the property that for any uncountable subset $X \subseteq E$ we have $\{\Phi(x, y): x, y \in X, x \neq y\}=k$. Such a space we call fullangled. The value of this notion is the following: We show that from the existence of a full-angled space it follows that a space of the same dimension exists which has no $\perp$-dense orthogonal system. Thus we get a variant of a result in $\S 1$. In addition we show, by using a set-theoretic idea of S. Todorčević, that a full-angled space of dimension $\aleph_{1}$ can be constructed in ZFC.

In view of our results in $\$ 2$ the question arises not only whether $\mathrm{CH}$ is necessary for the existence of uncountable-dimensional $(* *)$-spaces over finite or countable fields, but rather whether under $p=\aleph_{1}$ such spaces always exist. In $\S 4$ we show that under the condition $b=\aleph_{1}$ there exist always $\aleph_{1}$-dimensional $(* *)$-spaces over a special class of countable fields. Here $\mathfrak{b}$ is another cardinal invariant. It is known that $\mathfrak{p} \leq \mathfrak{b}$, so $\mathfrak{b}=\aleph_{1}$ is stronger than $\mathfrak{p}=\aleph_{1}$. By recent results of the second author (see [S2]), this result cannot be improved for $\mathfrak{p}$ instead of $b$ : Nonexistence of $(* *)$ spaces is consistent with $\mathfrak{p}=\aleph_{1}$ as well as is their existence with $b>\aleph_{1}$.

We remark that the main results of this paper are true in a rather more general context than that of symmetric bilinear forms. What is needed essentially is that the form defines a symmetric orthogonality relation. So we can generalize to orthosymmetric sesquilinear forms. But then, using a representation theorem (for AClattices equipped with a polarity) the main results can be transferred to the level of abstract ortho-lattices (see [G1] for the definitions). This has been announced in [S3].

Much of this material appeared originally in [S1].

§o. The cardinal invariants $b$ and $p$. With $\omega$ several cardinals can be associated by ignoring finite sets (see [vD]). They are uncountable and $\leq c$. In ZFC it cannot be decided where they lie exactly. In this paper, two of them play an important role. To define them we need some terminology.

Define the quasi-order (reflexive, transitive relation) $\leq^{*}$ on ${ }^{\omega} \omega$ by

$$
f \leq^{*} g: \leftrightarrow f(n) \leq g(n) \text { for all but finitely many } n<\omega .
$$

A subset of ${ }^{\omega} \omega$ is called $\leq^{*}$-unbounded if it is unbounded in $\left({ }^{\omega} \omega, \leq^{*}\right)$.

Define a quasi-order $\subseteq^{*}$ on $\mathscr{P}(\omega)$ by

$$
F \subseteq * G: \leftrightarrow n \in G \text { for all but finitely many } n \in F .
$$

A subset $A$ of $\omega$ is called a pseudointersection of a family $\mathscr{F}$ in $\mathscr{P}(\omega)$ if $\forall F \in$ $\mathscr{F}\left(A \subseteq^{*} F\right)$. We say that $\mathscr{F}$ has the strong f.i.p. if every nonempty finite subfamily has infinite intersection.

$\mathfrak{b}$ and $\mathfrak{p}$ are defined as follows:

$\mathfrak{b}=\min \left\{|B|: B\right.$ is $\leq^{*}$-unbounded in $\left.{ }^{\omega} \omega\right\}$.

$\mathfrak{p}=\min \{|\mathscr{F}|: \mathscr{F}$ is a family in $\mathscr{P}(\omega)$ with the strong f.i.p. which has no infinite pseudointersection $\}$. 
We quote parts of Theorems 3.1, 5.1 and 5.3 in [vD].

THEOREM 1. (a) $\mathfrak{\aleph}_{1} \leq \mathfrak{p} \leq \mathrm{b} \leq \mathrm{c}$.

(b) $b$ and $p$ are regular.

THEOREM 2. Let $\kappa$ and $\lambda$ be regular cardinals with $\aleph_{1} \leq \kappa \leq \lambda$. It is consistent with $Z F C$ that $\mathfrak{c}=\lambda$ and $\mathrm{b}=\mathfrak{p}=\kappa$.

THEOREM 3. It is consistent with ZFC that $\mathfrak{b}>\mathfrak{p}$.

In case $\kappa=\lambda$ Theorem 2 follows from MA, by means of the following theorem which is 2.15 in [ $\mathrm{Ku}$ ]. We also quote 2.18 and 2.19 in [ $\mathrm{Ku}$ ], which we need in $\S 1$. For Corollary 3 , refer to $[\mathrm{F}]$.

THEOREM $4(\mathrm{MA}(\kappa))$. Let $\mathscr{A}, \mathscr{C} \subseteq \mathscr{P}(\omega)$, where $|\mathscr{A}| \leq \kappa,|\mathscr{C}| \leq \kappa$, and assume that, for all $C \in \mathscr{C}$ and all finite $\mathscr{M} \subseteq \mathscr{A},|C-\bigcup \mathscr{M}|=\omega$; then there is $D \subseteq \omega$ such that $\forall A \in \mathscr{A}(|D \cap A|<\omega)$ and $\forall C \in \mathscr{C}(|D \cap C|=\omega)$.

Corollary 1 (MA). $\forall \kappa<\mathfrak{c}\left(2^{\kappa}=\mathfrak{c}\right)$.

COROLlaRY 2 (MA). c is regular.

Corollary 3 (MA). $\mathfrak{p}=\mathrm{c}$.

\$1. The paper of Baur and Gross under Martin's axiom instead of the continuum hypothesis. By assuming Martin's axiom instead of the continuum hypothesis we generalize all the results in $[B / G]$. The crucial step lies in generalizing their Lemma 1 , which allows us to define a form as desired by transfinite recursion. The constructions can then be done in an analogous way.

By $k$ we always denote a finite or countable field. We consider symmetric bilinear forms $\Phi: E \times E \rightarrow k$, where $E$ is a vector space over $k$. Most of the spaces we construct will have isotropic vectors, i.e. nonzero vectors $x$ such that $\Phi(x, x)$ $=0$. For a subspace $U \subseteq E$ the orthogonal complement $U^{\perp}$ is the subspace $\{x \in E$ : $\forall y \in U(\Phi(x, y)=0)\}$. We call $U \perp$-dense iff $U^{\perp \perp}=E$. If $(E, \Phi)$ is nondegenerate, which means $E^{\perp}=\{0\}$, then $U$ is $\perp$-dense iff $U^{\perp}=\{0\}$. Finally, $U$ is called diagonal iff it is spanned by an orthogonal basis.

\subsection{Extending forms.}

LEMMA $1(\mathrm{MA}(\kappa))$. Let $E$ be a hyperplane in the $\kappa$-dimensional $k$-vectorspace $E_{1}$ and $\left(U_{1}\right)_{1<\kappa}$ a family of subspaces of $E$ with $\operatorname{dim} U_{1}=\aleph_{0}$. Assume that $\Phi$ is a nondegenerate form on $E$. Then there exists a nondegenerate extension $\Phi_{1}$ of $\Phi$ to $E_{1}$ such that for all $l<\kappa$ the orthogonal complement of $U_{1}$ in $E_{1}$ is contained in $E$.

PROOF. Let $\left(e_{\alpha}\right)_{\alpha<\kappa}$ be a basis of $E$, and let $e$ span a linear supplement of $E$ in $E_{1}$.

1) We consider the following partially ordered set:

$P=\left\{\phi:\left[\bigoplus_{\alpha \in A_{\phi}} k e_{\alpha} \oplus k e\right]^{2} \rightarrow k: A_{\phi} \subset \kappa\right.$ is finite, $\phi$ is a symmetric bilinear form and $\phi|\operatorname{dom} \phi \cap \operatorname{dom} \Phi=\Phi| \operatorname{dom} \phi \cap \operatorname{dom} \Phi\}$

ordered by $\phi \leq \psi: \leftrightarrow \phi \subseteq \psi$.

We show that $(P, \leq)$ satisfies ccc: Assume that there is an uncountable antichain $\mathscr{A} \subseteq P$. By the $\Delta$-system lemma, applied to $\left\{A_{\phi}: \phi \in \mathscr{A}\right\}$, there exists a finite $W \subset \kappa$ and an uncountable $\mathscr{A}_{1} \subseteq \mathscr{A}$ such that $\forall \phi, \psi \in \mathscr{A}_{1}\left(A_{\phi} \cap A_{\psi}=W\right)$. Because $k$ is finite or countable there exists an uncountable $\mathscr{A}_{2} \subseteq \mathscr{A}_{1}$ such that

$$
\forall \phi, \psi \in \mathscr{A}_{2}\left(\phi\left|\left[\bigoplus_{\alpha \in W} k e_{\alpha} \oplus k e\right]^{2}=\psi\right|\left[\bigoplus_{\alpha \in W} k e_{\alpha} \oplus k e\right]^{2}\right) .
$$

Then all $\phi, \psi \in \mathscr{A}_{2}$ are compatible. This is a contradiction. 
2) Let $(\langle x(v), l(v)\rangle)_{v<\kappa}$ be an enumeration of $E \times \kappa$. For $v<\kappa$ let $D_{v}=\{\phi \in P$ : $x(v) \in \bigoplus_{\alpha \in A_{\phi}} k e_{\alpha}$ and there exists $u \in U_{u(v)} \cap \bigoplus_{\alpha \in A_{\phi}} k e_{\alpha}$ such that $\left.\phi(u, x(v)+e) \neq 0\right\}$. The sets $D_{v}$ are dense in $P$. For let $v<\kappa$ and $\phi \in P$. Choose $u \in U_{l(v)}-\bigoplus_{\alpha \in A_{\phi}} k e_{\alpha}$. Then $u$ can be written as

$$
u=\sum_{\alpha \in A_{\phi}} \lambda_{\alpha} e_{\alpha}+\sum_{\alpha \in B} \lambda_{\alpha} e_{\alpha}
$$

where $B \subset \kappa$ is finite and not empty, $B \cap A_{\phi}=\varnothing$ and $\forall \alpha \in B\left(\lambda_{\alpha} \neq 0\right)$. Similarly,

$$
x(v)=\sum_{\alpha \in A_{\phi}} \xi_{\alpha} e_{\alpha}+\sum_{\alpha \in B^{\prime}} \xi_{\alpha} e_{\alpha}
$$

where $B^{\prime} \subset \kappa$ is finite and $B^{\prime} \cap A_{\phi}=\varnothing$.

We can certainly assign elements of $k$ as values of $\psi\left(e_{\alpha}, e\right), \alpha \in B \cup B^{\prime}$, such that

$$
\sum_{\alpha \in A_{\phi} \cup B, \beta \in A_{\phi} \cup B^{\prime}} \lambda_{\alpha} \xi_{\beta} \Phi\left(e_{\alpha}, e_{\beta}\right)+\sum_{\alpha \in A_{\phi}} \lambda_{\alpha} \phi\left(e_{\alpha}, e\right)+\sum_{\alpha \in B} \lambda_{\alpha} \psi\left(e_{\alpha}, e\right) \neq 0 .
$$

We complete the definition of $\psi$ so that $\psi \in P$, dom $\psi=\left[\bigoplus_{\alpha \in A_{\phi} \cup B \cup B^{\prime}} k e_{\alpha} \oplus k e\right]^{2}$ and $\psi$ extends $\phi$. Thus $\psi \in D_{v}$.

3) By $\operatorname{MA}(\kappa)$ there exists a filter $F \subseteq P$ such that $\forall v<\kappa\left(F \cap D_{v} \neq \varnothing\right)$. Thus $\Phi_{1}:=\bigcup F$ is a well-defined symmetric bilinear form from $E_{1} \times E_{1}$ into $k$.

Now let $x+\lambda e \in E_{1}, \lambda \neq 0$ and $l<\kappa$. There exists $v<\kappa$ such that $\langle x(v), \imath(v)\rangle=$ $\left\langle\frac{1}{\lambda} x, l\right\rangle$. $\Phi_{1}$ extends a $\phi \in D_{v}$. By the definition of $D_{v}$ there exists $u \in U_{1}$ such that $\Phi_{1}(u, x+\lambda e) \neq 0$. We conclude that $U_{\imath}^{\perp_{\Phi_{1}}} \subseteq E$. It is clear now that $\Phi_{1}$ is nondegenerate.

THEOREM 1 (MA). There exists a nondegenerate form $\Phi$ on a $k$-vectorspace $E$ of dimension $c$ such that for all infinite-dimensional subspaces $U$ of $E$ we have $\operatorname{dim} U^{\perp}<$ c.

ProOF. Pick some $k$-space $E=\bigoplus_{\alpha<_{c}} k e_{\alpha}$ and let $\mathscr{U}=\left(U_{t}\right)_{\omega \leq l<c}$ be an enumeration of all $\aleph_{0}$-dimensional subspaces of $E$ (there are $(\mathfrak{c} \cdot|k|)^{\omega}=\mathfrak{c}$ of them). For all $\alpha<\mathfrak{c}$ we set $E_{\alpha}=\bigoplus_{\beta<\alpha} k e_{\beta}$. We can always renumber $\mathscr{U}$ so that $\forall \omega \leq \alpha<$ $\mathrm{c}\left(U_{\alpha} \subseteq E_{\alpha}\right)$.

We start on $E_{\omega}$ with some arbitrary nondegenerate form $\Phi_{\omega}$. Assume that $\Phi_{\alpha}$ is defined on $E_{\alpha}$ for $\omega \leq \alpha<\mathrm{c}$. We extend $\Phi_{\alpha}$ to $\Phi_{\alpha+1}$ on $E_{\alpha+1}=E_{\alpha} \oplus k e_{\alpha}$ by means of the lemma, where now $\left(U_{\beta}\right)_{\omega \leq \beta<\alpha}$ plays the role of $\left(U_{t}\right)_{i<x}$. If $\lambda<\mathrm{c}$ is a limit, let $\Phi_{\lambda}=\bigcup_{\omega \leq \alpha<\lambda} \Phi_{\alpha}$. In this way we get a form $\Phi$ on $E$ with the desired properties.

Remark. From Theorem 1 in $\S 2$ and Corollary 3 in $\S 0$ it follows that under $\neg \mathrm{CH}$ the space in Theorem 1 cannot have property (**).

1.2. Some remarkable properties of the space of Theorem 1. For a space to satisfy the assertion of Theorem 2 and its corollary in [G/O, pp. 516-517], it suffices to satisfy $(*)$ and, additionally, to preserve $(*)$ under algebraic extensions of the base field. It is not difficult to modify Lemma 1 in a way such that the space in Theorem 1 is like that.

Another modification of Lemma 1 says that if $k$ is an ordered field and $\Phi$ is positive definite, then it is possible to arrange for $\Phi_{1}$ to be positive definite as well. The proof is done analogously. Let $P^{\prime}$ be the subset of elements of $P$ that are positive definite, ordered by the restricted order, and let $D_{v}^{\prime}=D_{v} \cap P^{\prime}$. One shows that all 
$D_{v}^{\prime}$ are dense in $P^{\prime}$ and proceeds similarly. Thus, as in [B/G, p. 493], we get the following two theorems.

A vectorspace automorphism $T$ of the $k$-space $E$ is called locally algebraic if for each $x \in E$ there is some polynomial $f_{x} \in k[X]$ such that $f_{x}(T) x=0$; if $f_{x}$ does not depend on $x$ we call $T$ algebraic.

THEOREM 2. If $(E, \Phi)$ is as in Theorem 1 , then the set $\mathscr{A}$ of all locally algebraic isometries is a group. A coincides with the set of all algebraic isometries on $E$; furthermore, $\mathscr{A}$ is generated by -1 and the symmetries about nondegenerate $(\perp-$ closed) hyperplanes of E. In particular, $\mathscr{A}$ is a normal subgroup of the orthogonal group of $E$.

THEOREM 3. If in Theorem 1 we let $k$ be ordered, then $\Phi$ can be required to be positive definite. For such a space the set $\mathscr{L}_{s}=\left\{U \subseteq E: U+U^{\perp}=E\right\}$ of splitting subspaces, ordered by inclusion, is a lattice (in fact a modular lattice).

1.3. A space which admits no $\perp$-dense orthogonal family. In $[B / G]$ there was constructed an $\aleph_{1}$-dimensional space such that for any subspace $U$ which is spanned by an orthogonal basis we have $U^{\perp} \neq\{0\}$. For this Jensen's principle $\diamond$ (which implies $\mathrm{CH}$ ) had to be assumed. Some time later Gross [G2] realized that their construction can be done under $\mathrm{CH}$ only. His method of proof can be combined with our generalized Lemma 1. Thus under MA we get a space as above, i.e., without $\perp$-dense diagonal $U$, that is of dimension c.

We do not know whether the existence of such spaces of dimension $c$ is independent of ZFC. But in $\$ 3$ we shall construct in ZFC such a space of dimension $\aleph_{1}$.

By Corollary 2 in $\S 0$, MA implies that $c$ is regular. Thus under MA we get the following version of Lemma 2 in $[B / G]$.

Lemma 2 (MA). Let $(E, \Phi)$ be of dimension $c$ and let $\left(E_{\alpha}\right)_{\alpha<c}$ be an increasing sequence of subspaces of $E$ such that $\forall \alpha<\mathfrak{c}\left(\operatorname{dim} E_{\alpha}<\mathrm{c}\right), E=\bigcup_{\alpha<c} E_{\alpha}$, and $E_{\alpha}=$ $\bigcup_{\beta<\alpha} E_{\beta}$ if $\alpha$ is a limit. If $U$ is a subspace spanned by a basis $\left(u_{i}\right)_{\imath<c}$ and with $U^{\perp}=\{0\}$, then the set $A(U)=\left\{\alpha<\mathrm{c}: \alpha\right.$ is a limit ordinal, $U \cap E_{\alpha}=\bigoplus_{u_{\imath} \in E_{\alpha}} k u_{\imath}$ and $\left.\left(U \cap E_{\alpha}\right)^{\perp} \cap E_{\alpha}=\{0\}\right\}$ is both closed and unbounded in $\mathrm{c}$.

THEOREM 4 (MA). There exists a nondegenerate space $(E, \Phi)$ of dimension c such that every subspace $U$ spanned by an orthogonal basis has $U^{\perp} \neq\{0\}$.

Proof. Let $E=\bigoplus_{\imath}<c k e_{\imath} \oplus k e_{\imath}^{\prime}$, and for each $\omega \leq \alpha<c$ let $E_{\alpha}=\bigoplus_{\imath<\alpha} k e_{\imath} \oplus k e_{\imath}^{\prime}$. Let $\mathscr{U}_{\alpha}=\left(U_{\alpha, \beta}\right)_{\beta<c}$ be an enumeration of all infinite-dimensional subspaces of $E_{\alpha}$. (There are $(\kappa \cdot \omega)^{\kappa}=2^{\kappa}$ of them, where $\kappa=|\alpha|$, and, by Corollary 1 of $\S 0$, MA implies $\forall \kappa<\mathfrak{c}\left(2^{\kappa}=\mathfrak{c}\right)$.)

Assume now that $\gamma<\mathrm{c}$ and for each $\alpha<\gamma$ a nondegenerate form $\Phi_{\alpha}$ has been defined on $E_{\alpha}$ such that $\Phi_{\alpha} \subset \Phi_{\beta}$ for $\alpha<\beta$. If $\gamma$ is a limit then let $\Phi_{\gamma}=\bigcup_{\alpha<\gamma} \Phi_{\alpha}$. If $\gamma=$ $\alpha+1$ we define $\Phi_{\gamma}$ on $E_{\gamma}=E_{\alpha} \oplus k e_{\alpha} \oplus k e_{\alpha}^{\prime}$ as follows: Extend $\Phi_{\alpha}$ to $\Phi_{\gamma} \mid\left[E_{\alpha} \oplus k e_{\alpha}\right]^{2}$ by means of Lemma 1, where $\left(U_{v, \mu}\right)_{\max \{v, \mu\}<\gamma}$ plays the role of $\left(U_{l}\right)_{l<\kappa}$. We can do this in such a way that $\Phi_{\gamma}\left(e_{\alpha}, e_{\alpha}\right)=0$. Then set $\Phi_{\gamma}\left(e_{\alpha}^{\prime}, e_{\alpha}^{\prime}\right)=0, \Phi_{\gamma}\left(e_{\alpha}, e_{\alpha}^{\prime}\right)=1$ and $\Phi_{\gamma}\left(x, e_{\alpha}^{\prime}\right)=0$ for all $x \in E_{\alpha}$. It is not difficult to see that $\Phi_{\gamma}$ is nondegenerate.

An orthogonal system whose linear span is $\perp$-dense must be of cardinality $c$. This follows from the construction of $\Phi$ and the regularity of $\mathfrak{c}$ under MA. Assume now that $\left(u_{\imath}\right)_{\imath<c}$ is such a family. By Lemma 2 , for each $\beta<\mathfrak{c}$ there exists $\alpha<\mathfrak{c}$ with $\beta \leq \alpha$ 
such that

$$
\begin{aligned}
& E_{\alpha} \cap \bigoplus_{i<c} k u_{i}=\bigoplus_{i<c} E_{\alpha} \cap k u_{i}, \\
& \left(E_{\alpha} \cap \bigoplus_{i<c} k u_{i}\right)^{\perp} \cap E_{\alpha}=\{0\} .
\end{aligned}
$$

We choose $\alpha_{0}<\mathrm{c}$ such that (1) and (2) hold for $\alpha_{0}$. There exists $\beta_{0}<\mathrm{c}$ such that $E_{\alpha_{0}} \cap \bigoplus_{l<c} k u_{l}=U_{\alpha_{0}, \beta_{0}}$. Next we choose $\alpha_{1}>\max \left\{\alpha_{0}, \beta_{0}\right\}$ such that (1) and (2) hold for $\alpha_{1}$. Because $\operatorname{dim} E_{\alpha_{1}}<\operatorname{dim} \bigoplus_{l<c} k u_{\imath}$, there exists $\imath_{0}<c$ such that $u_{t_{0}} \notin E_{\alpha_{1}}$. $u_{i_{0}}$ can be written as

$$
u_{\iota_{0}}=\sum_{\alpha \in J}\left(\xi_{\alpha} e_{\alpha}+\xi_{\alpha}^{\prime} e_{\alpha}^{\prime}\right)+w
$$

where $\forall \alpha \in J\left(\alpha \geq \alpha_{1}\right)$ and $w \in E_{\alpha_{1}}$. We set $v=\sum_{\alpha \in J} \xi_{\alpha} e_{\alpha}+w$. Because by (1) $U_{\alpha_{0}, \beta_{0}}$ is spanned by some of the $u_{1}, u_{t_{0}} \notin E_{\alpha_{1}}$ and $U_{\alpha_{0}, \beta_{0}} \subseteq E_{\alpha_{0}} \subset E_{\alpha_{1}}$, we conclude that $\forall u \in U_{\alpha_{0}, \beta_{0}}\left(0=\Phi\left(u, u_{t_{0}}\right)=\Phi(u, v)\right)$. In other words, $v \in U_{\alpha_{0}, \beta_{0}}^{\perp}$. Thus by construction $v=w$. We get $u_{\imath_{0}}=\sum_{\alpha \in J} \xi_{\alpha}^{\prime} e_{\alpha}^{\prime}+w$. By (1), for $\alpha_{1}$ we have

$$
\forall u \in E_{\alpha_{1}} \cap \bigoplus_{i<c} k u_{i}\left(0=\Phi\left(u_{t_{0}}, u\right)=\Phi(w, u)\right)
$$

thus $w \in\left(E_{\alpha_{1}} \cap \bigoplus_{\imath<c} k u_{\imath}\right)^{\perp} \cap E_{\alpha_{1}}=\{0\}$ by (2). We conclude that

$$
u_{i_{0}}=\sum_{\alpha \in J} \xi_{\alpha}^{\prime} e_{\alpha}^{\prime}
$$

then $u_{t_{0}}$ is isotropic and therefore $u_{t_{0}} \in\left(\oplus_{\imath<c} u_{\imath}\right)^{\perp}$. This is a contradiction.

\section{§2. Independence proofs.}

2.1. The existence of $(* *)$-spaces is independent of ZFC. We show that the existence of $(* *)$-spaces of uncountable dimension over finite or countable fields is not only consistent with ZFC (as shown by Theorem 1 of $\$ 1$ ) under $\mathrm{CH}$, but even independent of $\mathrm{ZFC}$.

THEOREM $1\left(\mathfrak{p}>\aleph_{1}\right)$. Let $(E, \Phi)$ be an uncountable-dimensional space over a finite or countable field $k$. Then there exists an infinite-dimensional subspace $U \subseteq E$ with $\operatorname{dim} U^{\perp}>\aleph_{0}$. Thus $(E, \Phi)$ does not have the property $(* *)$.

In fact we can get more: Let $U$ be an infinite-dimensional subspace of $E$ and $X$ a set of $\aleph_{1}$ vectors in $E$. Then there are infinite-dimensional subspaces $U_{0} \supseteq U_{1} \supseteq \cdots \supseteq$ $U_{n} \supseteq \cdots$ of $U$ so that $\forall x \in X \exists n\left(x \in U_{n}^{\perp}\right)$. Hence, in particular, if $U$ and $V$ are subspaces of $E$ with $\operatorname{dim} U \geq \aleph_{0}$ and $\operatorname{dim} V>\aleph_{0}$, then there is an infinite-dimensional $U^{\prime} \subseteq U$ such that $\operatorname{dim} U^{\perp} \cap V>\aleph_{0}$.

Proof. Suppose $U$ and $X$ are given. For each $x \in X$ let $F_{x}=\{u \in U: \Phi(u, x)=0\}$. Let $\left(u_{n}\right)_{n<\omega}$ be a basis of $U$, and for each $n<\omega$ let $G_{n}$ be the subspace spanned by $\left\{u_{m}: m \geq n\right\}$. Then $\left\{F_{x}: x \in X\right\} \cup\left\{G_{n}: n<\omega\right\}$ has the strong f.i.p.: In order to see that $F_{x_{1}} \cap F_{x_{2}} \cap \cdots \cap F_{x_{r}} \cap G_{n}$ is infinite (even infinite dimensional), just note that it suffices to find $\lambda_{1}, \ldots, \lambda_{r+1} \in k$ for every $m<\omega$ such that $\forall 1 \leq j \leq r$

$$
\Phi\left(\sum_{i=1}^{r+1} \lambda_{i} u_{n+m+i}, x_{j}\right)=0,
$$


and this amounts to solving $r$ simultaneous homogeneous equations for the $r+1$ variables $\lambda_{1}, \ldots, \lambda_{r+1}$. So there is a nontrivial solution.

By $\mathfrak{p}>\aleph_{1}$ and countability of $U$ there exists an infinite $A=\left\{a_{n}: n<\omega\right\} \subseteq U$ such that $A-F_{x}$ and $A-G_{n}$ are finite for all $x \in X$ and $n<\omega$. Hence the subspace spanned by $A$ is infinite dimensional. But now if $U_{n}$ is the subspace spanned by $\left\{a_{m}: m \geq n\right\}$ it is not difficult to see that $\forall x \in X \exists n\left(x \in U_{n}^{\perp}\right)$, as desired.

2.2. (*)- and (**)-spaces and partitions of cardinals. The way we became aware of Theorem 1 is as follows. Let $(E, \Phi)$ be a space of uncountable dimension $\kappa$ over a finite or countable field $k$. Let $\left(e_{\alpha}\right)_{\alpha<\kappa}$ be a basis of $E$ and $k=\left\{\lambda_{n}: n \in N\right\}$, where $|N| \leq \aleph_{0}$. For $\alpha<\kappa$ and $n \in N$ we set

$$
A_{\alpha n}=\left\{\beta<\kappa: \Phi\left(e_{\alpha}, e_{\beta}\right)=\lambda_{n}\right\}
$$

We get a matrix

$$
\left[\begin{array}{cccc}
A_{00} & \cdots & A_{0 n} & \cdots \\
\vdots & & \vdots & \\
A_{\alpha 0} & \cdots & A_{\alpha n} & \cdots \\
\vdots & & \vdots &
\end{array}\right]
$$

with $\kappa$ rows and $|N|$ columns. By definition, for each $\alpha<\kappa$ the sets in the $\alpha$ th row partition $\kappa$.

Now let $\left(\left\langle\alpha_{i}, n_{i}\right\rangle\right)_{i<\omega}$ be a sequence in $\kappa \times N$ such that $\forall i, i^{\prime} \in \omega\left(i \neq i^{\prime} \rightarrow \alpha_{i} \neq \alpha_{i^{\prime}}\right)$. Set $A=\bigcap_{i<\omega} A_{\alpha_{i} n_{i}}$. Suppose that $\beta, \beta^{\prime} \in A$. Then we have for all $i<\omega$

$$
\Phi\left(e_{\alpha_{i}}, e_{\beta}-e_{\beta^{\prime}}\right)=\Phi\left(e_{\alpha_{i}}, e_{\beta}\right)-\Phi\left(e_{\alpha_{i}}, e_{\beta^{\prime}}\right)=\lambda_{n_{i}}-\lambda_{n_{i}}=0,
$$

or in other words

$$
\sum_{\beta, \beta^{\prime} \in A} k\left(e_{\beta}-e_{\beta^{\prime}}\right) \subseteq\left(\bigoplus_{i<\omega} k e_{\alpha_{i}}\right)^{\perp} .
$$

Hence, if there exists a model in which for any matrix as above there exists a sequence $\left(\left\langle\alpha_{i}, n_{i}\right\rangle\right)_{i<\omega}$ so that the set $A$ is uncountable or even has cardinality $\kappa$, then (**)- or $(*)$-spaces, respectively, of dimension $\kappa$ cannot exist. In case $N$ is finite such a model exists, as we see by the following theorem, which has been proved independently by Magidor in [Ma]. Thus, we get another proof of Theorem 1 for finite fields. Lemma 1 in $\S 4$ shows that the version of Theorem 2 in case $N$ is infinite is not consistent.

THEOREM $2\left(\mathfrak{p}>\mathfrak{\aleph}_{1}\right)$. Let $\omega_{1}=A_{i 1} \dot{\cup} A_{i 2} \dot{\cup} \cdots \dot{\cup} A_{\text {in }}, i<\omega$, be infinitely many partitions of $\omega_{1}$. Then there exists an infinite $N \subseteq \omega$ and $m \in\{1, \ldots, n\}$ such that $\bigcap_{i \in N} A_{\text {im }}$ is uncountable.

ProOF. Let $\mathscr{U}$ be a nonprincipal ultrafilter on $\omega$. For each $\alpha<\omega_{1}$ and $1 \leq j \leq n$ set $Y_{\alpha j}=\left\{i<\omega: \alpha \in A_{i j}\right\}$. Then we have $\omega=Y_{\alpha 1} \dot{\cup} \cdots \dot{\cup} Y_{\alpha n}$. Hence, for every $\alpha$ there exists a unique $j_{\alpha}$ such that $Y_{\alpha j_{\alpha}}$ belongs to $\mathscr{U}$. Choose $m \leq n$ such that $j_{\alpha}=m$ for uncountably many $\alpha$, and let $\mathscr{F}=\left\{Y_{\alpha j_{x}}: j_{\alpha}=m\right\}$. Then $\mathscr{F}$ has the strong f.i.p. and has cardinality $\aleph_{1}$. By $\mathfrak{p}>\aleph_{1}, \mathscr{F}$ has an infinite pseudointersection $A$. Thus for every $Y_{\alpha m} \in \mathscr{F}$ there exists $n_{\alpha}<\omega$ such that $A-n_{\alpha} \subseteq Y_{\alpha m}$. If we choose an uncountable $M \subseteq \omega_{1}$ and $n<\omega$ so that $\forall \alpha \in M\left(A-n \subseteq Y_{\alpha m}\right)$, then by definition $M \subseteq \bigcap_{i \in A-n} A_{i m}$. 
Conversely, by Theorem 1 of $\S 1$, in case of the field $k=\{0,1\}$, we get the following fact.

THeOREM 3 (MA). There exist partitions $\mathrm{c}=A_{\alpha} \dot{\cup} B_{\alpha}(\alpha<\mathrm{c})$ such that for each infinite $M \subset \mathrm{c}$ we have $\left|\bigcap_{\alpha \in M} A_{\alpha}\right|<\mathrm{c}$ and $\left|\bigcap_{\alpha \in M} B_{\alpha}\right|<\mathrm{c}$.

Proof. Let $A_{\alpha}=\left\{\beta<\mathfrak{c}: \Phi\left(e_{\alpha}, e_{\beta}\right)=0\right\}$ and $B_{\alpha}=\left\{\beta<\mathfrak{c}: \Phi\left(e_{\alpha}, e_{\beta}\right)=1\right\}$, where $\Phi$ is the form of Theorem 1 in $\S 1$, and $\left(e_{\alpha}\right)_{\alpha<c}$ is a basis of the space.

By Theorem 2, Theorem 3 is independent if $c=\aleph_{1}$. If $c>\aleph_{1}$ this is also true. Namely, Theorem 3 fails if $\mathrm{cf}(\mathfrak{c})>\omega_{1}$ and the statement | (a simplified version of $\&$ due to Tall) holds, which is consistent with $\neg \mathrm{CH}$ by unpublished (to our knowledge) results of Shelah and the first author. We give Shelah's proof.

THEOREM 4. For any uncountable cardinal $\kappa$, the statement

$$
\mid: \exists \mathscr{S} \subseteq\left[\omega_{1}\right]^{\omega}\left(|\mathscr{S}|=\omega_{1} \wedge \forall X \subseteq \omega_{1}\left(|X|=\omega_{1} \rightarrow \mathscr{P}(X) \cap \mathscr{S} \neq \varnothing\right)\right)
$$

is consistent with $\mathrm{c}=\kappa$.

Proof. Assume that $\mathrm{CH}$ holds in the ground model. Let $P$ be the notion of forcing for adding $\kappa$ Cohen subsets of $\omega_{1}$ with countable conditions, and let $Q$ be the notion of forcing for collapsing $\omega_{1}$ onto $\omega$ with finite conditions. We force with $P \times Q$. Now $\omega_{1}^{V^{P \times Q}}=\omega_{2}^{V}$. It will suffice to show that if $X$ is cofinal in $\omega_{2}^{V}, X \in V^{P \times Q}$, then some infinite subset of $X$ lies in $V$. Now since $V^{P} \models|Q|=\omega_{1}$ there must be $Y \subseteq X, Y$ cofinal in $\omega_{2}^{V}, Y \in V^{P}$. But $V^{P}$ and $V$ have the same countable sets of ordinals. So we are done.

THEOREM 5. If the statement $\mid$ holds and $\operatorname{cf}(\mathrm{c})>\omega_{1}$, then for every family of $\boldsymbol{\aleph}_{1}$ many partitions $\mathfrak{c}=A_{\alpha} \dot{\cup} B_{\alpha}\left(\alpha<\omega_{1}\right)$ there exists an infinite $M \subseteq \mathfrak{c}$ such that either $\bigcap_{\alpha \in M} A_{\alpha}$ or $\bigcap_{\alpha \in M} B_{\alpha}$ has cardinality $\mathrm{c}$.

Proof. For every $\imath<c$ let $X_{\imath}=\left\{\alpha<\omega_{1}: \imath \in A_{\alpha}\right\}$ and $Y_{\imath}=\left\{\alpha<\omega_{1}: l \in B_{\alpha}\right\}$. Clearly we have $\omega_{1}=X_{1} \dot{\cup} Y_{i}$. Hence, without loss of generality we may assume that $\mathrm{c}$ many $X_{\imath}$ are uncountable. By $\mid$, for any such $X_{\imath}$ there exists $S_{\imath} \in \mathscr{S}$ such that $S_{\imath} \subset X_{\imath}$. By $|\mathscr{S}|=\omega_{1}$ and $\operatorname{cf}(\mathrm{c})>\omega_{1}$ we conclude that there exist $M \in \mathscr{S}$ and $A \subseteq \mathrm{c}$ with $|A|=c$ such that $\forall i \in A\left(S_{\imath}=M\right)$ and hence $A \subseteq \bigcap_{\alpha \in M} A_{\alpha}$.

2.3. The existence of $(*)$-spaces of dimension $c$ is independent of ZFC. We show that by countable-support Sacks forcing over a model of $\mathrm{GCH}$ we get a model for $\left(\mathfrak{b}=\mathfrak{p}=\boldsymbol{N}_{1}\right)+\neg \mathrm{CH}$ where $(*)$-spaces of dimension $\mathfrak{c}$ cannot exist. We call a function $f: \omega \rightarrow \omega$ weakly increasing if (a) $f(0) \geq 1$, (b) $\forall m \leq n(f(m) \leq f(n))$ and (c) $\forall m \exists n(f(n) \geq m)$. Then it is a well-known combinatorial fact about this sort of forcing that if $G$ is generic over the ground model $V$, then in the extension $V[G]$ the following holds:

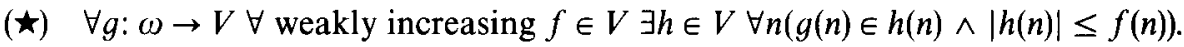

THEOREM 6. If $\mathrm{CH}$ holds in $V$ and we adjoin $\kappa$ Sacks reals with countable support, where $\operatorname{cf}(\kappa)>\omega_{1}$, then in the extension, the following holds: If $(E, \Phi)$ is a space of dimension $\kappa$ over a finite or countable field $k$, then for any infinite-dimensional subspace $U$ of $E$ there exists an infinite-dimensional $U^{\prime} \subseteq U$ such that $\operatorname{dim} U^{\prime \perp}=\kappa$.

Proof. We may suppose $\operatorname{dim} U=\aleph_{0}$. Let $\mathscr{B}$ be a countable basis for $U$ and let $\left(B_{n}\right)_{n<\omega}$ be a partition of $\mathscr{B}$ into finite sets such that $\left|B_{n}\right|=n+2$. Say $B_{n}=\left\{b_{i}^{n}: i<\right.$ $n+2\}$. Now let $\left(e_{\alpha}\right)_{\alpha<\kappa}$ be a basis for $E$. For $\alpha<\kappa$, let $g_{\alpha}(n)=\left\langle a_{0}^{n}, \ldots, a_{n+1}^{n}\right\rangle$, 
where $a_{i}^{n}=\Phi\left(b_{i}^{n}, e_{\alpha}\right)$. We may certainly assume that the underlying set of $k$ is an ordinal $\leq \omega$. Hence the $g_{\alpha}$ map into $V$.

Let $f(n)=n+1$. Then $f$ is weakly increasing and $f \in V$. So by $(\star)$ there is $h_{\alpha} \in V$ such that $\forall n\left(g_{\alpha}(n) \in h_{\alpha}(n) \wedge\left|h_{\alpha}(n)\right| \leq f(n)=n+1\right)$. Since $\mathrm{CH}$ holds in $V$ there are only $\aleph_{1}$ different $h_{\alpha}$ 's (we may assume that $h_{\alpha}(n)$ consists of $(n+2)$-tuples of integers), and since $\operatorname{cf}(\kappa)>\omega_{1}$ there is $X \subseteq \kappa$ and $h$ such that $|X|=\kappa$ and $\forall \alpha \in$ $X\left(h_{\alpha}=h\right)$.

Fix $n$, and let $h(n)=\left\{\left\langle\alpha_{i j}^{n}: j<n+2\right\rangle: i<n+1\right\}$. Now it is easy to find $\beta_{0}^{n}, \ldots, \beta_{n+1}^{n} \in k$ such that $\sum_{j=0}^{n+1} \beta_{j}^{n} \alpha_{i j}^{n}=0$ for $i=0, \ldots, n$ (this is just solving a homogeneous system of $n+1$ equations in $n+2$ unknowns), and not all $\beta_{i}^{n}$ are 0 . Let $u_{n}=\sum_{j=0}^{n+1} \beta_{j}^{n} b_{j}^{n}$. Then $\forall \alpha \in X\left(\Phi\left(e_{\alpha}, u_{n}\right)=0\right)$. So, if $U^{\prime}$ is the span of $\left\{u_{n}: n<\omega\right\}$, we conclude that

$$
\bigoplus_{\alpha \in X} k e_{\alpha} \subseteq U^{\prime \perp}
$$

REMARK. Using ( $\star$ ), it is not difficult to see that in the model of Theorem 6 we have $\mathfrak{b}=\mathfrak{p}=\aleph_{1}$. By $\S 4$, under $\mathfrak{b}=\aleph_{1}$ there always exists a (*)-space of dimension $\aleph_{1}$. Hence the following question remains open:

QUESTION. Does there exist a model where there are no $(*)$-spaces?

§3. Full-angled spaces. Up to now the question of whether the continuum hypothesis is necessary for the existence of $(* *)$-spaces has remained open. We shall now provide a negative answer. We introduce the notion of a full-angled space, by means of which we shall prove a variant of Theorem 4 in $\$ 1$, and construct in ZFC an $\aleph_{1}$-dimensional space that admits no $\perp$-dense orthogonal system.

Let $k$ be a finite or countable field and $(E, \Phi)$ a symmetric bilinear space of uncountable dimension over $k$. We call $(E, \Phi)$ full-angled, if for any uncountable set $X \subseteq E$ we have $\{\Phi(x, y): x, y \in X, x \neq y\}=k$. Note that if $(E, \Phi)$ is full-angled then there can be no uncountable set of mutually orthogonal vectors. In particular, if $(E, \Phi)$ is full-angled then there exists a nondegenerate full-angled space of the same dimension (take a linear supplement of $E^{\perp}$, equipped with the restricted form).

\subsection{Existence of full-angled spaces and consequences.}

THEOREM 1. If $(E, \Phi)$ is full-angled and nondegenerate, then there exists a nondegenerate space $\left(E_{1}, \Phi_{1}\right)$ of the same dimension such that for every subspace $U \subseteq E_{1}$, if $U$ has an orthogonal basis, then $U^{\perp} \neq\{0\}$.

PROOF. It is not difficult to find a basis $\left(e_{\alpha}\right)_{\alpha<\kappa}$ for $E$ such that, for each $x \in E$, $\left\{e_{\alpha}: \Phi\left(e_{\alpha}, x\right) \neq 0\right\}$ is infinite. Let $F$ be a disjoint space with basis $\left\{f_{\alpha}: \alpha<\kappa\right\}$ and set $E_{1}=E \oplus F$. We extend $\Phi$ to $E_{1}$ by defining $\Phi_{1}\left(f_{\alpha}, f_{\beta}\right)=0$ for all $\alpha$ and $\beta$, $\Phi_{1}\left(e_{\alpha}, f_{\beta}\right)=0$ if $\alpha \neq \beta$, and $\Phi_{1}\left(e_{\alpha}, f_{\alpha}\right)=1$. Then $\Phi_{1}$ is still nondegenerate. For let $x=\lambda_{1} e_{\alpha_{1}}+\cdots+\lambda_{m} e_{\alpha_{m}}+\mu_{1} f_{\beta_{1}}+\cdots+\mu_{n} f_{\beta_{n}} \in E_{1}$. If $m=0$ then $\Phi_{1}\left(x, e_{\beta_{1}}\right)=$ $\mu_{1} \neq 0$, while if $m>0$ then $\Phi_{1}\left(x, e_{\alpha}\right) \neq 0$, where $\alpha$ is chosen so that $\Phi_{1}\left(\lambda_{1} e_{\alpha_{1}}+\cdots+\right.$ $\left.\lambda_{m} e_{\alpha_{m}}, e_{\alpha}\right) \neq 0$ and $\alpha \neq \beta_{1}, \ldots, \beta_{n}$.

First assume that $U$ is a subspace such that the set $A(U)=\left\{\alpha<\kappa: e_{\alpha}\right.$ appears in the representation of some $x \in U\}$ is countable. If we choose $\alpha \in \kappa-A(U)$ then clearly $f_{\alpha} \in U^{\perp}$.

Hence it suffices to see that any $U$ with uncountable $A(U)$ cannot have an orthogonal basis. Suppose not. 
If $x \in E$ let $x=\lambda_{1} e_{\alpha_{1}}+\cdots+\lambda_{n} e_{\alpha_{n}}, \lambda_{1}, \ldots, \lambda_{n} \neq 0$, and define $M(x)=\left\{\alpha_{1}, \ldots, \alpha_{n}\right\}$. Define $M(y)$ similarly for $y \in F$.

By hypothesis, we may find a linearly independent orthogonal family $\left(u_{\alpha}\right)_{\alpha<\omega_{1}}$ in $U$ such that for $\alpha<\beta$ we have $\max M\left(x_{\alpha}\right)<\max M\left(x_{\beta}\right)$. By the $\Delta$-system lemma and countability of $k$ we may find an uncountable $A \subseteq \omega_{1}$ such that $u_{\alpha}=v+$ $x_{\alpha}+y_{\alpha}$, where $x_{\alpha} \in E$ and $y_{\alpha} \in F,\left(M\left(x_{\alpha}\right) \cup M\left(y_{\alpha}\right)\right) \cap\left(M\left(x_{\beta}\right) \cup M\left(y_{\beta}\right)\right)=\varnothing$ and $\Phi\left(v, x_{\alpha}+y_{\alpha}\right)$ has the same value, say $\lambda$, for all $\alpha, \beta \in A, \alpha \neq \beta$. Note that $x_{\alpha} \neq 0$ and $\Phi\left(x_{\alpha}+y_{\alpha}, x_{\beta}+y_{\beta}\right)=\Phi\left(x_{\alpha}, x_{\beta}\right)$ for $\alpha, \beta \in A, \alpha \neq \beta$, and thus

$$
\Phi\left(u_{\alpha}, u_{\beta}\right)=\Phi(v, v)+2 \lambda+\Phi\left(x_{\alpha}, x_{\beta}\right) .
$$

However, since $(E, \Phi)$ is full-angled we may find $\alpha, \beta \in A$ so that $\Phi\left(u_{\alpha}, u_{\beta}\right)=$ $\Phi(v, v)+2 \lambda+\Phi\left(x_{\alpha}, x_{\beta}\right) \neq 0$, and this contradicts the assumption that the $u_{\alpha}$ were orthogonal.

The following theorem shows that the existence of a full-angled space of dimension $\mathrm{c}$ is consistent and that there are models for $\mathrm{ZFC}+\neg \mathrm{CH}$ where (**)-spaces exist.

THEOREM 2. Suppose we force by adding $\kappa$ Cohen reals, where $\kappa>\aleph_{0}$. Let $E$ be $a \kappa$-dimensional vector space over a finite or countable field $k$ in the extension. Then there exists a nondegenerate symmetric bilinear form $\Phi$ on $E$ such that, for any countable linearly independent set $X \subseteq E$, for all but countably many vectors $y \in E$ it is true that $\{\Phi(x, y): x \in X\}=k$. Hence, in particular, $(E, \Phi)$ is full-angled and, for any infinite-dimensional $U \subseteq E, \operatorname{dim} U^{\perp} \leq \aleph_{0}$.

Proof. Usually the forcing conditions for adding $\kappa$ Cohen reals are taken to be finite functions from subsets of $\kappa$ into $\omega$ (or 2 or any $n \geq 2$ ). Let $\mathscr{B}=\left\{e_{\alpha}: \alpha<\kappa\right\}$ be a basis for $E$. It is fully equivalent to add $\kappa$ Cohen reals with finite functions from $\mathscr{B} \times \mathscr{B}$ into $k$ subject to the requirement that if $p$ is a condition then $p\left(e_{\alpha}, e_{\beta}\right)=$ $p\left(e_{\beta}, e_{\alpha}\right)$ always. Thus the forcing determines a symmetric bilinear form $\Phi$ on $E$, and an easy argument with genericity shows that $\Phi$ is nondegenerate. (We assume $k$ is in the ground model, which is legitimate by the homogeneity of Cohen forcing.)

Suppose $X \subseteq E$ is countably infinite and linearly independent. Then there exists a countable-dimensional subspace $U$ spanned by vectors from $\mathscr{B}$ so that $X \subseteq U$. Suppose $y \in E-U$. Say $y=\sum_{i=1}^{m} \lambda_{i} e_{\alpha_{i}}$, where $e_{\alpha_{1}} \notin U$ and each $\lambda_{i} \neq 0$. Given any condition $p$, there must be an $x \in X$ such that $x=\sum_{i=1}^{n} \mu_{i} e_{\beta_{i}}$ and $p$ assigns no value yet to $\left\langle e_{\alpha_{1}}, e_{\beta_{1}}\right\rangle$. (We use the assumption that $X$ is infinite and linearly independent here.) Extend $p$ arbitrarily to all pairs $\left\langle e_{\alpha_{i}}, e_{\beta_{i}}\right\rangle$ except for $\left\langle e_{\alpha_{1}}, e_{\beta_{1}}\right\rangle$. Fix $\lambda \in k$. It is now easy to define $p$ on $\left\langle e_{\alpha_{1}}, e_{\beta_{1}}\right\rangle$ to ensure that $\Phi(x, y)=\lambda$. Thus by genericity we must have $\Phi(x, y)=\lambda$ for some $x \in X$.

As announced above, from Theorems 1 and 2 we get the following corollaries:

COROLLARY 1. It is consistent with ZFC that $\mathrm{c}$ is large and that for any finite or countable field $k$ there exists a nondegenerate space $(E, \Phi)$ over $k$ of dimension $\mathrm{c}$ with the $(* *)$-property.

COROLLARY 2. It is consistent with $Z F C$ that $\mathrm{c}$ is large and that for any finite or countable field $k$ there exists a nondegenerate space $(E, \Phi)$ over $k$ of dimension $\mathrm{c}$ so that for any diagonal $U \subseteq E$ we have $U^{\perp} \neq\{0\}$.

3.2 Construction of a full-angled space in ZFC. In ZFC we construct a fullangled space of dimension $\aleph_{1}$. We use the following set-theoretical proposition 
which is inspired by a variation due to Velleman of Todorčević's proof that $\aleph_{1} \nrightarrow\left[\aleph_{1}\right]_{\aleph_{1}}^{2}$ (see $[\mathrm{T}]$ ).

THEOREM 3. Let $\left[\omega_{1}\right]^{2}=\left\{\{\alpha, \beta\}: \alpha, \beta \in \omega_{1}, \alpha \neq \beta\right\}$. There exists a function $f$ : $\left[\omega_{1}\right]^{2} \rightarrow \omega$ such that for any collection $\left\{F_{\alpha}: \alpha<\omega_{1}\right\}$ of disjoint nonempty finite subsets of $\omega_{1}$ there are $n<\omega$ and a function $t: n \times n-\{\langle n-1, n-1\rangle\} \rightarrow \omega$ such that for all $m<\omega$ there are $\alpha$ and $\beta, \alpha \neq \beta$, such that, if $F_{\alpha}=\left\{\alpha_{0}, \ldots, \alpha_{n-1}\right\}$ and $F_{\beta}=\left\{\beta_{0}, \ldots, \beta_{n-1}\right\}$, then for each $\langle i, j\rangle \in n \times n,\langle i, j\rangle \neq\langle n-1, n-1\rangle$, we have $f\left\{\alpha_{i}, \beta_{j}\right\}=t\langle i, j\rangle$, while $f\left\{\alpha_{n-1}, \beta_{n-1}\right\}=m$.

ProOF. Fix a decomposition $\left\{S_{n}: n<\omega\right\}$ of $\omega_{1}$ into disjoint stationary sets. Let $\left\{s_{\alpha}: \alpha<\omega_{1}\right\}$ be a collection of distinct countably infinite sequences of zeros and ones. Let $(\langle\sigma(i), m(i)\rangle: i\langle\omega)$ enumerate all pairs $\langle\sigma, m\rangle$ where $\sigma$ is a finite sequence of zeros and ones and $m<\omega$. For each $\alpha<\omega_{1}, \alpha \neq 0$, let $h_{\alpha}: \omega \rightarrow \alpha$ be onto.

We begin by defining $g:\left[\omega_{1}\right]^{2} \rightarrow \omega_{1}$. Suppose $\alpha<\beta<\omega_{1}$. Let $i$ be minimal such that $s_{\alpha}(i) \neq s_{\beta}(i)$. Let $F=\left\{h_{\beta}(j): j \leq i\right\}$. If $\{\gamma \in F: \alpha \leq \gamma\}$ is nonempty, let $g\{\alpha, \beta\}$ be the least element of this set; otherwise let $g\{\alpha, \beta\}=0$.

Now suppose $g\{\alpha, \beta\} \in S_{i}$. If $\sigma(i) \subseteq s_{\alpha}$ and $\sigma(i) \subseteq s_{\beta}$, then set $f\{\alpha, \beta\}=m(i)$; otherwise set $f\{\alpha, \beta\}=0$. We claim that $f$ is as desired.

Let $\left\{F_{\alpha}: \alpha<\omega_{1}\right\}$ consist of pairwise disjoint nonempty finite subsets of $\omega_{1}$. Without loss of generality we may assume $\left|F_{\alpha}\right|=n$ for all $\alpha$. We will write $F_{\alpha}=$ $\left\{\alpha_{0}, \ldots, \alpha_{n-1}\right\}, F_{\beta}=\left\{\beta_{0}, \ldots, \beta_{n-1}\right\}$, and so on.

Fix $\alpha<\omega_{1}$. Choose $p_{\alpha}<\omega$ large enough so that the $s_{\alpha_{i}} \mid p_{\alpha}$ are all distinct. Let $G_{\alpha}=\bigcup_{i<n}\left\{h_{\alpha_{i}}(j): j \leq p_{\alpha}\right\} \cap \alpha$. Then $G_{\alpha}$ is a finite subset of $\alpha$. By Fodor's theorem there is a stationary set $Z \subseteq \omega_{1}$ so that for some $p, G$ and $\left\langle\tau_{0}, \ldots, \tau_{n-1}\right\rangle$ we have $p_{\alpha}=p, G_{\alpha}=G$ and $\forall i<n\left(s_{\alpha_{i}} \mid p=\tau_{i}\right)$, for every $\alpha \in Z$. If we choose $\left\{F_{\alpha}^{\prime}: \alpha<\omega_{1}\right\}$ so that for all $\alpha<\omega_{1}$ we have $F_{\alpha}^{\prime}=F_{\gamma}$ for some $\gamma \geq \alpha, \gamma \in Z$, then it is clear that for every $\alpha>\max G$ we have $p_{\alpha}=p, G_{\alpha}=G$ and $\forall i<\omega\left(s_{\alpha_{i}} \mid p=\tau_{i}\right)$ for these new $F_{\alpha}^{\prime}$. Thus we may as well assume that the original $F_{\alpha}$ have this property. In addition, without loss of generality we may omit the countably many $F_{\alpha}$ with either $\alpha \leq \max G$ or $F_{\alpha} \cap(\max G)+1 \neq \varnothing$.

Next we aim at obtaining $t$. Fix $\alpha<\omega_{1}$. Define $t_{\alpha}: n \times n-\{\langle n-1, n-1\rangle\} \rightarrow$ $\omega$ as follows: Let $t_{\alpha}\langle i, i\rangle=0$ for $i<n-1$. Suppose $i, j<n, i \neq j$. First we define $g_{\alpha}\langle i, j\rangle\left\langle\omega_{1}\right.$. Let $q$ be minimal so that $\tau_{i}(q) \neq \tau_{j}(q)$; and let $F=\left\{h_{\alpha_{j}}(r): r \leq q\right\}$. If $\{\gamma \in F: \alpha \leq \gamma\}$ is nonempty let $g_{\alpha}\langle i, j\rangle$ be the least element of this set; otherwise let $g_{\alpha}\langle i, j\rangle=0$.

Now suppose $g_{\alpha}\langle i, j\rangle \in S_{r}$. If $\sigma(r) \subseteq \tau_{i}$ and $\sigma(r) \subseteq \tau_{j}$, then set $t_{\alpha}(i, j)=m(r)$; otherwise set $t_{\alpha}\langle i, j\rangle=0$.

Note that if $i \neq j$ and $\alpha_{i}<\min \left\{\beta, \beta_{j}\right\}$, then we have $g\left\{\alpha_{i}, \beta_{i}\right\}=g_{\beta}\langle i, j\rangle$ and hence $f\left\{\alpha_{i}, \beta_{j}\right\}=t_{\beta}\langle i, j\rangle$.

Since there are only countably many distinct functions $t_{\alpha}$, one of them must occur uncountably often. Call it $t$.

Let $C_{0}=\left\{\alpha\right.$ : if $\beta<\alpha$ then $F_{\beta} \subseteq \alpha$ and if $\beta \geq \alpha$ then $\left.F_{\beta} \cap \alpha=\varnothing\right\}$. Then $C_{0}$ is closed unbounded. Suppose we are given finite sequences $\sigma_{0}, \ldots, \sigma_{n-1}$. Let $X=$ $\left\{\alpha: \forall i<n\left(\sigma_{i} \subset s_{\alpha_{i}}\right)\right\}$. If $X$ is uncountable let $C\left(\sigma_{0}, \ldots, \sigma_{n-1}\right)$ be the set of limit points of $X$; otherwise let $C\left(\sigma_{0}, \ldots, \sigma_{n-1}\right)$ be any closed unbounded set beginning above sup $X$. Then $C_{0}$ and all the $C\left(\sigma_{0}, \ldots, \sigma_{n-1}\right)$ are countably many closed unbounded subsets of $\omega_{1}$; so the intersection $C$ of all of them is also closed unbounded. 
Now let $m<\omega$ be arbitrary. Find $l$ so that $\sigma(l)=\tau_{n-1}$ and $m(l)=m$. Let $\bar{\alpha} \in C \cap S_{l}, \bar{\alpha}>\max G$. (Recall that $S_{l}$ has been chosen stationary.) Choose $\beta>\bar{\alpha}$ such that $t_{\beta}=t$ and $\bar{\alpha} \notin F_{\beta}$. We will show that for some $\alpha<\bar{\alpha}$, the pair $\alpha, \beta$ satisfies the conclusion of the theorem.

First choose $p^{\prime} \geq p$ so large that for each $i<n$ there is some $j \leq p^{\prime}$ so that $h_{\beta_{i}}(j)=\bar{\alpha}$. (Here we use the condition $\bar{\alpha} \in C_{0}$.) For $i<n$ let $\sigma_{i}=s_{\beta_{i}} \mid p^{\prime}$. Then $X=$ $\left\{\gamma: \forall i<n\left(\sigma_{i} \subset s_{\gamma_{i}}\right)\right\}$ is uncountable, since $\beta \in X$ and $\beta>\bar{\alpha}, \bar{\alpha} \in C \subseteq C\left(\sigma_{0}, \ldots, \sigma_{n-1}\right)$. For each $\gamma \in X, \gamma \neq \beta$, there must be some $q_{\gamma}>p^{\prime}$ so that $\forall i<n\left(s_{\gamma_{i}}\left|q_{\gamma} \neq s_{\beta_{i}}\right| q_{\gamma}\right)$. Hence there are $q$ and $\rho_{0}, \ldots, \rho_{n-1}$ so that $Y=\left\{\gamma: q_{\gamma}=q\right.$ and $\left.\forall i<n\left(\rho_{i}=s_{\gamma_{i}} \mid q\right)\right\}$ is uncountable. It follows that since $\bar{\alpha} \in C \subseteq C\left(\rho_{0}, \ldots, \rho_{n-1}\right), \bar{\alpha}$ is a limit point of $Y^{\prime}=\left\{\gamma: \forall i<n\left(\rho_{i}=s_{\gamma_{i}} \mid q\right)\right\}$.

Choose $\alpha \in Y^{\prime}$ such that $\alpha<\bar{\alpha}$ and $\alpha>\sup \bigcup_{i<n}\left\{h_{\beta_{i}}(j): j \leq q\right\} \cap \bar{\alpha}$. We claim that $\alpha$ and $\beta$ are as desired. We have already checked that if $i, j<n$ and $i \neq j$, then $f\left\{\alpha_{i}, \beta_{j}\right\}=t\langle i, j\rangle\left(\right.$ since $\left.\alpha_{i}<\bar{\alpha}<\min \left\{\beta, \beta_{j}\right\}\right)$. Suppose now that $i<n$. Then it is easy to see that $g\left\{\alpha_{i}, \beta_{i}\right\}=\bar{\alpha}$. Since $\bar{\alpha} \in S_{l}$ and $\sigma(l)=\tau_{n-1}$, we see that $\sigma(l) \subset s_{\alpha_{i}}$ and $\sigma(l) \subset s_{\beta_{i}}$ iff $i=n-1$. Since $m(l)=m$, it follows from the definition of $f$ that $f\left\{\alpha_{i}, \beta_{i}\right\}=0$ if $i<n-1$, and $f\{n-1, n-1\}=m$. This completes the proof.

THEOREM 4. Let $k$ be a finite or countable field. Then there exists a full-angled $k$-space $(E, \Phi)$ of dimension $\aleph_{1}$.

Proof. In order to derive Theorem 4 from Theorem 3, let $\pi: \omega \rightarrow k$ be onto and let $\left(e_{\alpha}\right)_{\alpha<\omega_{1}}$ be a basis for $E$. Define $\Phi$ by setting

$$
\Phi\left(e_{\alpha}, e_{\beta}\right)=\pi \circ f\{\alpha, \beta\}
$$

if $\alpha \neq \beta$. $\Phi\left(e_{\alpha}, e_{\alpha}\right)$ may be defined arbitrarily.

Suppose $\left\{\boldsymbol{u}_{\alpha}: \alpha<\omega_{1}\right\} \subseteq E$. Using the notation of the proof of Theorem 1, we may assume $u_{\alpha}=v+x_{\alpha}$, where the sets $M\left(x_{\alpha}\right)$ are all nonempty and pairwise disjoint. Note that it suffices to see that $\left\{\Phi\left(x_{\alpha}, x_{\beta}\right): \alpha, \beta<\omega_{1}, \alpha \neq \beta\right\}=k$. By the countability of $k$ we may assume that there is $n<\omega$ and $\lambda_{0}, \ldots, \lambda_{n-1} \in k$ such that $\left|M\left(x_{\alpha}\right)\right|=n$ for all $\alpha<\omega_{1}$, and if $M\left(x_{\alpha}\right)=\left\{\alpha_{0}, \ldots, \alpha_{n-1}\right\}$ then $x_{\alpha}=\sum_{i=0}^{n-1} \lambda_{i} e_{\alpha_{i}}$.

Now find $t: n \times n-\{\langle n-1, n-1\rangle\} \rightarrow \omega$ as in Theorem 3 ( $n$, of course, is already determined). But now, for $\alpha$ and $\beta$ as in the conclusion of Theorem 3, we have

$$
\begin{aligned}
\Phi\left(x_{\alpha}, x_{\beta}\right) & =\sum_{i, j<n} \lambda_{i} \lambda_{j} \Phi\left(e_{\alpha_{i}}, e_{\beta_{j}}\right) \\
& =\sum_{\substack{i, j<n \\
(i, j) \neq(n-1, n-1)}} \lambda_{i} \lambda_{j} \pi \circ t\langle i, j\rangle+\lambda_{n-1}^{2} \pi \circ f\left\{\alpha_{n-1}, \beta_{n-1}\right\} .
\end{aligned}
$$

Since we may find $\alpha$ and $\beta$ as above such that $f\left(\left\{\alpha_{n-1}, \beta_{n-1}\right\}\right)$ is arbitrary in $\omega$, and hence $\lambda_{n-1}^{2} \pi \circ f\left\{\alpha_{n-1}, \beta_{n-1}\right\}$ is arbitrary in $k$, we are done.

COROLlaRY 3 . Let $k$ be a finite or countable field. Then there exists a nondegenerate $k$-space $(E, \Phi)$ of dimension $\aleph_{1}$ such that for all diagonal subspaces $U \subseteq E$ we have $U^{\perp} \neq\{0\}$.

REMARK. It follows from the Erdős-Rado partition theorem $\left(2^{\aleph_{0}}\right)^{+} \rightarrow\left(\aleph_{1}\right)_{\aleph_{0}}^{2}$ that if $k$ is finite or countable and $(E, \Phi)$ is a $k$-space of dimension $>2^{\aleph_{0}}$, then $(E, \Phi)$ cannot be full-angled. This shows that Theorems 2 and 4 cannot be improved. 
§4. Construction of $\mathbf{a}(* *)$-space when $b=\aleph_{1}$. Theorem 1 of $\$ 2$ says that when $\mathfrak{p}>\aleph_{1}(* *)$-spaces (of uncountable dimension over finite or countable fields) cannot exist.

By recent results of the second author, it is consistent that $\mathfrak{p}=\aleph_{1}$ holds and (**)spaces do not exist. In [S2], for an arbitrary finite or countable field $k$ a ccc partial ordering $P^{k}$ is defined such that no quadratic space over $k$ which lies in the ground model $V$ is $(* *)$ in the extension $V^{P^{k}}$. Iterating this forcing so that all fields are taken into consideration, we obtain a model in which there exist no $(* *)$-spaces. By choosing $V$ appropriately we can make sure that $\mathfrak{p}=\aleph_{1}$ holds in the final extension.

In this section we show that under the stronger condition $\mathfrak{b}=\aleph_{1}$ (for $\mathfrak{p} \leq \mathfrak{b}$ ) (**)-spaces of dimension $\aleph_{1}$ always exist, at least over a special class of countable fields.

In [S2] it is proved that $\mathbf{b}>\boldsymbol{\aleph}_{1}$ is consistent with " $(* *)$-spaces exist over arbitrary finite or countable fields" by showing that forcing with the natural partial ordering to adjoin a dominating function preserves $(* *)$-spaces. Hence it is impossible to prove equivalence of " $(* *)$-spaces exist" with either $b=\aleph_{1}$ or $\mathfrak{p}=\aleph_{1}$.

\subsection{A variation of the Ulam matrix.}

LEMMA 1. There exists a matrix $\left(A_{\alpha n}\right)_{\omega \leq \alpha<\omega_{1}, n<\omega}$, consisting of subsets $A_{\alpha n} \subset \omega_{1}$, with the following properties, $\forall \omega \leq \alpha<\omega_{1}$ :

(1) $\omega_{1}-\alpha=\bigcup_{n<\omega} A_{\alpha n}$ (disjoint union).

(2) The set $\left\{n<\omega: A_{\alpha n}\right.$ is unbounded in $\left.\omega_{1}\right\}$ is infinite.

(3) For every infinite sequence $\left(\left\langle\alpha_{i}, n_{i}\right\rangle\right)_{i<\omega}$ in $\left(\omega_{1}-\omega\right) \times \omega$ the set $\bigcap_{i<\omega} A_{\alpha_{i} n_{i}}$ contains at most one element.

Proof. Let $\left(F_{\alpha}\right)_{\omega \leq \alpha<\omega_{1}}$ be a family of almost disjoint sets $F_{\alpha} \subset \omega$. By transfinite recursion we construct a family $\left(f_{\alpha}\right)_{\omega \leq \alpha<\omega_{1}}$ of functions with the following properties, $\forall \omega \leq \alpha<\omega_{1}$ :

(4) $f_{\alpha}: F_{\alpha} \rightarrow \alpha \cup\{\alpha\}$ is one-to-one and onto.

(5) $\forall \beta<\omega_{1} \forall n<\omega\left(\exists \beta^{\prime}<\omega_{1}\left(\beta^{\prime} \geq \beta\right) \wedge \exists n^{\prime}<\omega\left(n^{\prime} \in F_{\beta^{\prime}} \wedge n^{\prime} \geq n\right)\right)\left(f_{\beta^{\prime}}\left(n^{\prime}\right)=\alpha\right)$.

Suppose $\left(f_{\alpha}\right)_{\omega \leq \alpha<\beta}$ is defined for $\omega \leq \beta<\omega_{1}$ such that any $f_{\alpha}$ has (4). We extend this family to $\left(f_{\alpha}\right)_{\omega \leq \alpha<\gamma}$, where $\beta<\gamma<\omega_{1}$, as follows. In the infinite set $M=\left\{n<\omega:\left\{\alpha<\omega_{1}: n \in F_{\alpha}\right\}\right.$ is unbounded in $\left.\omega_{1}\right\}$ we choose an infinite sequence $\left(n_{i}\right)_{i<\omega}$. Let $\left(\beta_{i}\right)_{i<\omega}$ be an enumeration of $\beta$. For each $\langle i, j\rangle \in \omega^{2}$ we choose $\alpha_{i j} \in$ $\omega_{1}-\beta$ such that $n_{i} \in F_{\alpha i j}$ and $\alpha_{i j} \neq \alpha_{i^{\prime} j^{\prime}}$ for $\langle i, j\rangle \neq\left\langle i^{\prime}, j^{\prime}\right\rangle$. Set $\gamma=\sup \left\{\alpha_{i j}\right.$ : $\left.\langle i, j\rangle \in \omega^{2}\right\}$. Now let $\beta \leq \alpha<\gamma$. If $\alpha=\alpha_{i j}$ for $\langle i, j\rangle \in \omega^{2}$, we choose $f_{\alpha}$ with (4) and $f_{\alpha}\left(n_{i}\right)=\beta_{j}$. Otherwise, let $f_{\alpha}$ be arbitrary with (4).

Now we define, for all $\omega \leq \alpha<\omega_{1}$ and $n<\omega$,

$$
A_{\alpha n}=\left\{v<\omega_{1}: n \in F_{v} \wedge f_{v}(n)=\alpha\right\} .
$$

Then (1), (2) and (3) can be read off from (4) and (5).

4.2. Definition of the space and proof of $(* *)$. Let $k$ be an arbitrary finite or countable field. We adjoin $\aleph_{0}$ many transcendentals $t_{n}(n<\omega)$. Let $K=$ $k\left(t_{0}, t_{1}, \ldots, t_{n}, \ldots\right)$, and let $E=\bigoplus_{\omega \leq \alpha<\omega_{1}} K e_{\alpha}$ be a $K$-space of dimension $\aleph_{1}$. On $E$ we define a symmetric bilinear form $\Phi$ by means of the matrix $\left(A_{\alpha n}\right)_{\omega \leq \alpha<\omega_{1}, n<\omega}$ from Lemma 1. For $\omega \leq \alpha \leq \beta<\omega_{1}$ we set

$$
\Phi\left(e_{\alpha}, e_{\beta}\right)=t_{n}: \leftrightarrow \beta \in A_{\alpha n} .
$$


By Lemma 1 the $n$, as above, is well-defined. We denote it by $n(\beta, \alpha)$. Thus, we have $f_{\beta}(n(\beta, \alpha))=\alpha$.

Proposition 1. $(E, \Phi)$ is nondegenerate.

Proof. Let $x=\sum_{i=1}^{n} \lambda_{i} e_{\alpha_{i}} \in E, \forall i\left(\lambda_{i} \neq 0\right)$. By (2) we can choose $j<\omega$ so that $t_{j}$ does not appear in $\lambda_{i}(1 \leq i \leq n)$ and, additionally, $A_{\alpha_{1} j}$ is unbounded in $\omega_{1}$. Thus, there exists $\beta \in A_{\alpha_{1} j}$ so that $\forall 1 \leq i \leq n\left(\beta \geq \alpha_{i}\right)$. We conclude that

$$
\Phi\left(x, e_{\beta}\right)=\sum_{i=1}^{n} \lambda_{i} \Phi\left(e_{\alpha_{i}}, e_{\beta}\right)=\sum_{i=1}^{n} \lambda_{i} t_{n\left(\beta, \alpha_{i}\right)} \neq 0,
$$

for, by construction, $n\left(\beta, \alpha_{i}\right) \neq n\left(\beta, \alpha_{j}\right)$ if $i \neq j$.

Lemma 2. Let $\left\langle a_{1}, \ldots, a_{n}\right\rangle \in K^{n}-\{\langle 0, \ldots, 0\rangle\}$. Then the equation $a_{1} X_{1}+\cdots+$ $a_{n} X_{n}=0$ in $n$ unknowns $X_{i}$ has at most $n-1$ pairwise disjoint solution vectors $\left\langle t_{l_{1}}, \ldots, t_{l_{n}}\right\rangle$, each of them consisting of transcendentals $t_{l_{i}}$, so that $\forall 1 \leq i, j \leq n$ $\left(i \neq j \rightarrow l_{i} \neq l_{j}\right)$.

Proof. It suffices to see that

$$
\left|\begin{array}{ccc}
t_{11} & \cdots & t_{1 n} \\
\vdots & & \vdots \\
t_{n 1} & \cdots & t_{n n}
\end{array}\right| \neq 0 .
$$

Expand the determinant by, say, the first row. Then by the induction hypothesis the cofactors are all nonzero, and the transcendentals of the first row do not appear in them by hypothesis.

Proposition 2. Let $U=\bigoplus_{l<\omega} K f_{l}$ be a subspace of $E$ with the property that there exists $q<\omega$ such that all the $f_{l}$ can be represented by at most $q$ basis vectors $e_{\alpha}$. Then $\operatorname{dim} U^{1} \leq \aleph_{0}$.

Proof. Suppose there exists a linearly independent family $\left(u_{t}\right)_{l<\omega_{1}}$ so that $\forall l<\omega \forall l<\omega_{1}\left(\Phi\left(f_{l}, u_{l}\right)=0\right)$. We write

$$
f_{l}=a_{l 1} e_{v(l, 1)}+a_{l 2} e_{v(l, 2)}+\cdots+a_{l q} e_{v(l, q)},
$$

where $\forall l<\omega\left(\left\langle a_{l 1}, \ldots, a_{l q}\right\rangle \neq\langle 0, \ldots, 0\rangle\right)$. Without loss of generality we may assume that for distinct $l, m$ we have $\{v(l, 1), \ldots, v(l, q)\} \cap\{v(m, 1), \ldots, v(m, q)\}=\varnothing$. Otherwise find linear combinations of the $f_{l}$ with this property and consider the span of them. By the $\Delta$-system lemma and the countability of $K$ we may assume that there exist $x \in \bigoplus_{\alpha<\sup \{v(l, i): l<\omega, 1 \leq i \leq q\}} K e_{\alpha}, p<\omega$ and $\lambda_{1}, \ldots, \lambda_{p} \in K-\{0\}$ such that $\forall \imath<\omega_{1}$

(6) $u_{\imath}=x+\lambda_{1} e_{\alpha(i, 1)}+\lambda_{2} e_{\alpha(i, 2)}+\cdots+\lambda_{p} e_{\alpha(i, p)}$,

where, for all $l<\xi<\omega_{1}$, we have $\sup _{l<\omega, 1 \leq i \leq q} v(l, i) \leq \alpha(l, 1)<\cdots<\alpha(l, p)<$ $\alpha(\xi, 1)<\cdots<\alpha(\xi, p)$. By taking differences of vectors $u_{i}$ we can assume that in $(6)$ we have $x=0$. Thus $\forall k<\omega \forall l<\omega_{1}$

$$
\begin{aligned}
\Phi\left(f_{l}, u_{l}\right)= & a_{l 1} \lambda_{1} t_{n(\alpha(l, 1), v(l, 1))}+\cdots+a_{l 1} \lambda_{p} t_{n(\alpha(l, p), v(l, 1))} \\
& +a_{l 2} \lambda_{1} t_{n(\alpha(l, 1), v(l, 2))}+\cdots+a_{l 2} \lambda_{p} t_{n(\alpha(i, p), v(l, 2))} \\
\vdots & \vdots \\
& +a_{l q} \lambda_{1} t_{n(\alpha(l, 1), v(l, q))}+\cdots+a_{l q} \lambda_{p} t_{n(\alpha(l, p), v(l, q))}=0 .
\end{aligned}
$$


We claim that not even the first $p \cdot q$ vectors $u_{\imath}$ are in $U^{\perp}$. Because the sets $F_{\alpha}$ are chosen almost disjoint, we can find $l_{1}<\omega$ such that $\forall l<\omega\left(l \geq l_{1}\right) \forall 0 \leq l<\xi<p q$

$$
\begin{aligned}
& \{n(\alpha(l, i), v(l, j)): 1 \leq i \leq p, 1 \leq j \leq q\} \\
& \quad \cap\{n(\alpha(\xi, i), v(l, j)): 1 \leq i \leq p, 1 \leq j \leq q\}=\varnothing
\end{aligned}
$$

and, additionally, the sets $\{n(\alpha(l, i), v(l, j)): 1 \leq i \leq p, 1 \leq j \leq q\}$ contain $p q$ distinct elements. By (7), for all $l \geq l_{1}$ the equation

$$
\begin{array}{cc}
a_{l 1} \lambda_{1} X_{1} & +\cdots+a_{l 1} \lambda_{p} X_{p} \\
+a_{l 2} \lambda_{1} X_{p+1} & +\cdots+a_{l 2} \lambda_{p} X_{2 p} \\
\vdots & \vdots \\
+a_{l q} \lambda_{1} X_{(q-1) p+1} & +\cdots+a_{l q} \lambda_{p} X_{q p}=0
\end{array}
$$

then has as solutions $p q$ pairwise disjoint sequences, whereas each one consists of $p q$ distinct transcendentals. This contradicts Lemma 2.

The question remains open how big the orthogonals of subspaces $U=\bigoplus_{l<\omega} K f_{l}$ are if there does not exist an upper bound for the numbers of basis vectors $e_{\alpha}$ representing the $f_{l}$. We show that such orthogonals are small too if we require that the almost disjoint family $\left(F_{\alpha}\right)_{\omega \leq \alpha<\omega_{1}}$ that we used to define $\Phi$ satisfies an additional property. The existence of such a family is equivalent to the statement $b=\aleph_{1}$. In [Ma], M. Magidor gives essentially the same proof for this equivalence.

LEMMA 3. The following statements are equivalent:

(i) $\mathbf{b}=\aleph_{1}$.

(ii) There exists an almost disjoint family $\left(F_{\alpha}\right)_{x<\omega_{1}}$ in $\mathscr{P}(\omega)$ with the property that for each uncountable $A \subseteq \omega_{1}$ there exists an infinite $N \subseteq A$ and $n<\omega$ so that $\forall \alpha$, $\beta \in N\left(\alpha \neq \beta \rightarrow\left|F_{\alpha} \cap F_{\beta}\right| \leq n\right)$.

ProOF. (i) $\rightarrow$ (ii). Let $\left(f_{\alpha}\right)_{\alpha<\omega_{1}}$ be an unbounded family in $\left({ }^{\omega} \omega, \leq^{*}\right)$. We may assume that for $\alpha<\beta$ we have $\left(f_{\alpha}<^{*} f_{\beta}\right)$. Thus any uncountable subfamily of $\left(f_{\alpha}\right)_{\alpha<\omega_{1}}$ is unbounded too. For $n<\omega$ let $f_{\alpha} \mid n$ denote the restriction of $f_{\alpha}$ to $n$. For all $\alpha<\omega_{1}$ set $F_{\alpha}=\left\{f_{\alpha} \mid n: n<\omega\right\}$. Then $\left(F_{\alpha}\right)_{\alpha<\omega_{1}}$ is an almost disjoint family in the countable set of all finite sequences of natural numbers.

We claim that $\left(F_{\alpha}\right)_{\alpha<\omega_{1}}$ has the property from (ii). For let $A \subseteq \omega_{1}$ be uncountable. If for every $n<\omega$ the set $\left\{f_{\alpha}(n): \alpha \in A\right\}$ is finite we choose $g(n)<\omega$ such that $\forall \alpha \in$ $A\left(f_{\alpha}(n)<g(n)\right)$. Thus $g$ is an upper bound for $\left(f_{\alpha}\right)_{\alpha \in A}$. This is a contradiction. We conclude that there exists $n<\omega$ so that $\left\{f_{\alpha}(n): \alpha \in A\right\}$ is infinite. We choose an infinite $N \subseteq A$ such that the values $f_{\alpha}(n)$ for $\alpha \in N$ are pairwise distinct. Then we have $\forall \alpha, \beta \in N\left(\alpha \neq \beta \rightarrow\left|F_{\alpha} \cap F_{\beta}\right| \leq n\right)$.

(ii) $\rightarrow$ (i). Let $\left(F_{\alpha}\right)_{\alpha<\omega_{1}}$ be an almost disjoint family. For any $\alpha<\omega_{1}$ let $f_{\alpha}: \omega \rightarrow F_{\alpha}$ be an increasing enumeration of $F_{\alpha}$. Suppose $\mathfrak{b}>\aleph_{1}$. Then there exists $g \in{ }^{\omega} \omega$ so that $\forall \alpha<\omega_{1}\left(f_{\alpha}<* g\right)$. We conclude that there exists an uncountable $A \subseteq \omega_{1}$ and $n_{0}<\omega$ so that $\forall \alpha \in A \forall n \geq n_{0}\left(f_{\alpha}(n)<g(n)\right)$. Let $N \subseteq A$ be infinite. For all $\alpha \in N$ and $n \geq n_{0}$ we have $f_{\alpha}(0)<f_{\alpha}(1)<\cdots<f_{\alpha}(n)<g(n)$; thus $\left|F_{\alpha} \cap g(n)\right|>n$. Because $N$ is infinite we can choose $\alpha, \beta \in N, \alpha \neq \beta$, such that $F_{\alpha} \cap g(n)=F_{\beta} \cap g(n)$ and hence $\left|F_{\alpha} \cap F_{\beta}\right|>n$. We conclude that $\left(F_{\alpha}\right)_{\alpha<\omega_{1}}$ is not as in (ii). 
THEOREM. Let $\left(F_{\alpha}\right)_{\omega \leq \alpha<\omega_{1}}$ be an almost disjoint family of subsets $F_{\alpha} \subset \omega$ with the property that for each uncountable $A \subseteq \omega_{1}-\omega$ there exists an infinite $N \subseteq A$ and $n<\omega$ such that $\forall \alpha, \beta \in N\left(\alpha \neq \beta \rightarrow\left|F_{\alpha} \cap F_{\beta}\right| \leq n\right)$.

Let $(E, \Phi)$ be constructed as above by means of the family $\left(F_{\alpha}\right)_{\omega \leq \alpha<\omega_{1}}$. Then $(E, \Phi)$ has the property $(* *)$.

Proof. By Proposition 2 we need only look at subspaces $U=\bigoplus_{l<\omega} K f_{l}$ where the number of basis vectors $e_{\alpha}$ in the representations of each $f_{l}$ is unbounded. For $l<\omega$ let

$$
f_{l}=a_{l 1} e_{v(l, 1)}+a_{l 2} e_{v(l, 2)}+\cdots+a_{l n_{l}} e_{v\left(l, n_{l}\right)} .
$$

We may assume that the sequence $\left(n_{l}\right)_{l<\omega}$ is increasing and, for all $l<\omega$,

$$
\left\{v(l, i): 1 \leq i \leq n_{l}\right\} \nsubseteq \bigcup_{0 \leq r \leq l-1}\left\{v(r, i): 1 \leq i \leq n_{r}\right\} .
$$

Suppose there exists a linearly independent family $\left(u_{l}\right)_{l<\omega_{1}}$ such that $\forall l<\omega$ $\forall l<\omega_{1}\left(\Phi\left(f_{l}, u_{\imath}\right)=0\right)$. We may assume

$$
u_{t}=\lambda_{1} e_{\alpha(t, 1)}+\lambda_{2} e_{z(t, 2)}+\cdots+\lambda_{p} e_{\alpha(t, p)},
$$

where $p<\omega, \forall 1 \leq i \leq p\left(\lambda_{i} \in K-\{0\}\right)$, and for all $\imath<\xi<\omega_{1}$ we have

$$
\sup _{l<\omega, 1 \leq j \leq n_{l}} v(l, j) \leq \alpha(l, 1)<\cdots<\alpha(l, p)<\alpha(\xi, 1)<\cdots<\alpha(\xi, p) .
$$

Hence we calculate $\forall l<\omega \forall l<\omega_{1}$

$$
\begin{aligned}
\Phi\left(f_{l}, u_{l}\right)= & a_{l 1} \lambda_{1} t_{n(\alpha(l, 1), v(l, 1))}+\cdots+a_{l 1} \lambda_{p} t_{n(\alpha(l, p), v(l, 1))} \\
& +a_{l 2} \lambda_{1} t_{n(\alpha(l, 1), v(l, 2))}+\cdots+a_{l 2} \lambda_{p} t_{n(\alpha(l, p), v(l, 2))} \\
& \vdots \\
& +a_{l n_{l}} \lambda_{1} t_{n\left(\alpha(l, 1), v\left(l, n_{l}\right)\right)}+\cdots+a_{l n_{1}} \lambda_{p} t_{n\left(\alpha(l, p), v\left(l, n_{l}\right)\right)}=0 .
\end{aligned}
$$

By almost-disjointness of the $F_{\alpha(t, 1)}, \ldots, F_{\alpha(l, p)}$ and by (8) we find (perhaps after renumbering the $e_{v(l, j)}\left(1 \leq j \leq n_{l}\right)$ in $f_{l}$, which does not depend on $\left.l\right) l_{l}, m_{\imath}<\omega$ so that $\forall l<\omega\left(l \geq l_{\imath}\right)$

$$
\begin{aligned}
& \left\{n(\alpha(l, 1), v(l, j)): m_{l} \leq j \leq n_{l}\right\} \\
& \cap \bigcup_{2 \leq i \leq p}\left\{n(\alpha(l, i), v(l, j)): 1 \leq j \leq n_{l}\right\}=\varnothing .
\end{aligned}
$$

There exist an uncountable $A \subseteq \omega_{1}$ and $\bar{l}, \bar{m}<\omega$ so that $\forall \imath \in A\left(l_{l}=\bar{l} \wedge m_{\imath}=\bar{m}\right)$.

By (9) and (10), for all $l<\omega(l \geq \bar{l}), j<\omega\left(\bar{m} \leq j \leq n_{l}\right)$ and $l \in A$ the transcendental $t_{n(\alpha(l, 1), v(l, j))}$ must appear in one of the coefficients $a_{l i}\left(1 \leq i \leq n_{l}\right)$ or $\lambda_{i}(1 \leq i \leq p)(\star)$.

Now by hypothesis, there exists an infinite $N \subset A$ and $n<\omega$, so that

$$
\forall l, \xi \in N\left(l \neq \xi \rightarrow\left|F_{\alpha(l, 1)} \cap F_{\alpha(\xi, 1)}\right| \leq n\right) .
$$

Choose $l<\omega$ so that $l \geq \bar{l}$ and $n_{l}-\bar{m}>n$. Then the set

$$
\left\{n(\alpha(l, 1), v(l, j)): \bar{m} \leq j \leq n_{l}, l \in N\right\}
$$

is infinite. This contradicts our observation ( $\star$ ). 
Question. When $\mathfrak{b}=\aleph_{1}$, does there exist a $(* *)$-space over an arbitrary finite or countable field?

\section{REFERENCES}

[B/G] W. Baur and H. Gross, Strange inner product spaces, Commentarii Mathematici Helvetici, vol. 52 (1977), pp. 491-495.

[vD] E. K. VAN DòuWEN, The integers and topology, Handbook of set-theoretic topology (K. Kunen and J. E. Vaughan, editors), North-Holland, Amsterdam, 1984, pp. 111-167.

[F] D. H. Fremlin, Consequences of Martin's axiom, Cambridge University Press, Cambridge, 1984.

[G1] H. Gross, Quadratic forms in infinite dimensional vector spaces, Birkhäuser, Basel, 1979.

[G2] - Handwritten notes.

[G/O] H. Gross and E. OGG, Quadratic spaces with few isometries, Commentarii Mathematici Helvetici, vol. 48 (1973), pp. 511-519.

[Ku] K. KunEN, Set theory: an introduction to independence proofs, North-Holland, Amsterdam, 1980.

[Ma] M. MAGidor, Letter to Otmar Spinas, dated October 30, 1988.

[S1] O. SPINAS, Konsistenz- und Unabhängigkeitsresultate in der Theorie der quadratischen Formen, Ph.D. thesis, University of Zürich, Zürich, 1989.

[S2] - Iterated forcing in quadratic form theory (in preparation).

[S3] - An undecidability result in lattice theory, Abstracts of Papers Presented to the American Mathematical Society, vol. 11 (1990), p. 161.

[T] S. TodorčEvić, Partitioning pairs of countable ordinals, Acta Mathematica, vol. 159 (1987), pp. 261-294.

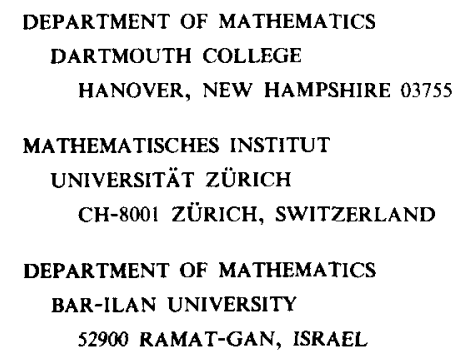

Sorting. The first address is Professor Baumgartner's; the other two are Professor Spinas' (Bar-Ilan University is the current one). 